\title{
A CLASS OF FINITE GROUPS HAVING NILPOTENT INJECTORS
}

\author{
M. J. IRANZO and F. PÉREZ MONASOR
}

(Received 19 February 1985; revised 10 April 1985)

\begin{abstract}
The purpose of this paper is to construct a class of groups which properly contains the class of $\mathscr{N}$-constrained groups, and which is such that all groups in this class have $\mathcal{N}$-injectors.
\end{abstract}

1980 Mathematics subject classification (Amer. Math. Soc.): 20 D 10.

All groups considered throughout this paper will be finite. We denote by $\mathscr{N}$ the class of nilpotent groups, by $\tilde{N}$ the class of quasinilpotent groups, i.e. $\tilde{\mathcal{N}}=$ $\left\{G \mid G=F(G) L(G)=F^{*}(G)\right\}$, and we put $\mathscr{L}=\left\{G \mid G=C_{G}(L(G)) L(G)\right\}$, where $L(G)$ is the semisimple radical of $G$ (the concept of semisimple group is taken from Gorenstein-Walter's paper [2]). The properties of the subgroups $F^{*}(G)$ and $L(G)$ which we shall use here are given in [3].

Let $\mathscr{M}$ be the class of all groups $G$ such that for every subnormal subgroup $N$ of $G$, we have $C_{N}(J) \leqslant J$, where $J$ is any $\mathcal{N}$-injector of $F^{*}(N)$. By [4] we know that every $\mathscr{N}$-injector of $F^{*}(G)$ constitutes the product of $F(G)$ and an $\mathscr{N}$-injector of $L(G)$.

A group $G$ is $\mathscr{N}$-constrained if $C_{G}(F(G)) \leqslant F(G)$. In $1971 \mathrm{~A}$. Mann proved that an $\mathscr{N}$-constrained group has an unique conjugacy class of $\mathscr{N}$-injectors. In [4] we proved that all groups belonging to $\mathscr{L}$ have $\mathscr{N}$-injectors. Using Lausch's theorem [5] we can obtain the following

TheOREM. Let $G$ be an $\mathscr{M}$-group. Then $G$ possesses $\mathscr{N}$-injectors, and these are exactly the $\mathcal{N}$-maximal subgroups of $G$ that contain the product of $F(G)$ and an N-injector of $L(G)$.

(C) 1986 Australian Mathematical Society $0263-6115 / 86 \$ A 2.00+0.00$ 
Progf. We use induction on $|G|$. Put $Z=Z(L(G))$. Let $G^{*}$ be a maximal normal subgroup of $G$. Then we have

$$
L(G) / Z=\left(L(G) \cap G^{*}\right) Z / Z \cdot R Z / Z,
$$

where $R \unlhd G$ is semisimple. Moreover, we have

$$
L(G) \cap G^{*}=\left(L(G) \cap G^{*}\right) Z\left(L(G) \cap G^{*}\right)=L\left(G^{*}\right) Z\left(L(G) \cap G^{*}\right) .
$$

On the other hand, $L(G)=\left(L(G) \cap G^{*}\right) R$, where $\left[L\left(G^{*}\right), R\right] \leqslant Z$, and by the three-subgroups lemma together with the perfectness of $L\left(G^{*}\right)$, it follows that $\left[L\left(G^{*}\right), R\right]=1$. Therefore $R \leqslant C_{L(G)}\left(L\left(G^{*}\right)\right)$, whence

$$
\left[R, G^{*}\right] \leqslant\left[C_{L(G)}\left(L\left(G^{*}\right)\right), G^{*}\right] \leqslant C_{L(G) \cap G^{*}}\left(L\left(G^{*}\right)\right)=Z\left(L(G) \cap G^{*}\right),
$$

and again by the three-subgroups lemma we obtain $\left[R, G^{*}\right]=1$. Now let $I$ be an $\mathcal{N}$-injector of $L(G)$. Then $I / Z$ is an $\mathcal{N}$-injector of $L(G) / Z$, so that $I / Z \cap R Z / Z$ is an $\mathcal{N}$-injector of $R Z / Z$, and $I / Z \cap L\left(G^{*}\right) Z / Z$ is an $\mathcal{N}$-injector of $L\left(G^{*}\right) Z / Z$. Thus we have

$$
I / Z=\left(I \cap L\left(G^{*}\right)\right) Z / Z \times(I \cap R) Z / Z,
$$

whence

$$
I=\left(I \cap L\left(G^{*}\right)\right)(I \cap R) Z=\left(I \cap L\left(G^{*}\right)\right)(I \cap R),
$$

because $Z=Z\left(L\left(G^{*}\right)\right) Z(R)$.

Now let $V$ be an $\mathscr{N}$-maximal subgroup of $G$ such that $I F(G) \leqslant V$, and suppose that $V \cap G^{*} \leqslant W \leqslant G^{*}$, where $W \in \mathscr{N}$. It is clear that $F\left(G^{*}\right) \leqslant V \cap$ $G^{*} \leqslant W \leqslant G^{*}$, and that $I \cap L\left(G^{*}\right) \leqslant V \cap G^{*} \leqslant W \leqslant G^{*}$. Thus

$$
I=\left(I \cap L\left(G^{*}\right)\right)(I \cap R) \leqslant\left(V \cap G^{*}\right)(I \cap R) \leqslant W(I \cap R) \leqslant G^{*}(I \cap R) .
$$

Moreover, $W(I \cap R) \in \mathscr{N}$ because $\left[G^{*}, R\right]=1$.

By the inductive hypothesis, we can assume that $W$ is an $\mathscr{N}$-injector of $G^{*}$. We now have two cases.

Case 1. $F(G) \leqslant G^{*}$. In this case $F\left(G^{*}\right)=F(G) \leqslant W$. Let $V_{1}$ be an $\mathscr{N}$-maximal subgroup of $G$ that contains $W(I \cap R)$. Then $I F(G) \leqslant V_{1}$. Since $C_{G}(I F(G))$ $\leqslant I F(G)$, an application of Lausch's theorem implies that there exists $g \in G$ such that $V_{1}=V^{g}$. In consequence, we have

$$
V \cap G^{*} \leqslant W \leqslant V_{1} \cap G^{*}=V^{g} \cap G^{*}=\left(V \cap G^{*}\right)^{g},
$$

and so $V \cap G^{*}=W$ is an $\mathscr{N}$-injector of $G^{*}$.

Case 2. $F(G) \nless G^{*}$. In this case $G=F(G) G^{*}$, whence $G / G^{*} \cong C_{p}$ for a prime number $p$. Thus, either $V G^{*} / G^{*}=1$, or $V G^{*}=G$. In the first case, $V G^{*}=G^{*}$, and so $F(G) \leqslant V \leqslant G^{*}$, which is a contradiction. Therefore, $V G^{*}=$ $G$. Now we have

$$
G / G^{*}=V G^{*} / G^{*} \cong V /\left(V \cap G^{*}\right) \cong C_{p}
$$


Since $V \cap W=V \cap G^{*}$, it follows that $V /(V \cap W) \cong C_{p}$, and since $F(G) \leqslant V$ but $F(G) \nless W$, we obtain

$$
V=F(G)(V \cap W)=V \cap F(G) W \leqslant F(G) W .
$$

Now, as $F(G) W$ is solvable, it possesses a unique conjugacy class of $\mathscr{N}$-injectors. If $W_{1}$ is one of them, then $W_{1}$ and $V$ are $\mathcal{N}$-maximal subgroups of $F(G) W$ containing $F(G)$; moreover, as $F(G) W / F(G)$ is nilpotent, it follows [1, Hilsatz 1] that $V$ and $W_{1}$ are conjugated in $F(G) W$. Hence $V$ is an $\mathcal{N}$-injector of $F(G) W$. Thus $V \cap G^{*}$ is an $\mathscr{N}$-maximal subgroup of $F(G) W \cap G^{*}=W\left(F(G) \cap G^{*}\right)=$ $W$, and so $V \cap G^{*}=W$.

Trivially, an $\mathcal{N}$-injector of $G$ is an $\mathscr{N}$-maximal subgroup of $G$ that contains the product of $F(G)$ and an $\mathscr{N}$-injector of $L(G)$.

Proposition 1. $\mathscr{L} \subset \mathscr{M}$.

Proof. Let $I$ be an $\mathcal{N}$-injector of $L(G)$, and let $G \in \mathscr{L}$. Then we have

$$
C_{G}(I F(G)) \leqslant C_{G}(I) \cap C_{G}(F(G))=C_{C_{G}(F(G))}(I) ;
$$

but $L(G) \leqslant C_{G}(F(G)) \leqslant L(G) C_{G}(L(G))$, and so

$$
\begin{aligned}
C_{G}(F(G)) & =L(G)\left(C_{G}(F(G)) \cap C_{G}(L(G))\right. \\
& \left.=L(G) C_{G}\left(F^{*}(G)\right)=L(G) Z\left(F^{*}(G)\right)\right) .
\end{aligned}
$$

From $Z\left(F^{*}(G)\right) \leqslant C_{C_{G}(F(G))}(I) \leqslant L(G) Z\left(F^{*}(G)\right)$ we get

$$
C_{C_{G}(F(G))}(I)=Z\left(F^{*}(G)\right) C_{L(G)}(I) \leqslant Z\left(F^{*}(G)\right) I=Z(F(G)) I .
$$

Now if $N$ is a subnormal subgroup of $G$, then since $\mathscr{L}$ is an $S_{n}$-closed class, $N$ is also an $\mathscr{L}$-group, and by the above argument we obtain $C_{N}(J) \leqslant J$, where $J$ is an $\mathscr{N}$-injector of $F^{*}(N)$. Thus $G$ is an $\mathscr{M}$-group.

ReMARK. The class $\mathscr{L}$ is properly contained in $\mathscr{M}$. In fact, if we take $G=A_{5}$ wr $C_{7}$, then $L(G)=A_{5}^{\#}$, i.e. the base group of $G$, and $C_{G}(L(G))=1$. Hence $G \notin \mathscr{L}$.

Moreover, we know that $A_{5}^{\#}$ is the unique minimal normal subgroup of $G$, whence all proper subnormal subgroups of $G$ are contained in $A_{5}^{\#}$. Let $N$ be one of them, so that $F^{*}(N)=N=L(N)$, and trivially $C_{N}(I) \leqslant I$ for every $\mathscr{N}$-injector $I$ of $N$. So it remains to prove that if $I$ is an $\mathscr{N}$-injector of $L(G)$, then

$$
C_{G}(I F(G)) \leqslant I F(G) \text {, i.e. } C_{G}(I) \leqslant I .
$$

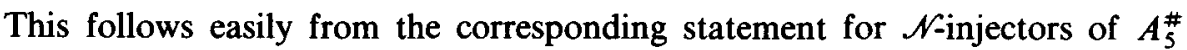
(which gives $\left.C_{L(G)}(I) \leqslant I\right)$ and from $C_{G}(I) \leqslant L(G)$ (which is obvious from the regular action of $C_{7} \leqslant G$ on $L(G)$ together with the fact that 7 does not divide $|L(G)|)$. 
If $S \leqslant G$, we put $[S]_{G}=\left\{S^{g} \mid g \in G\right\}$.

Proposition 2. If $G \in \mathscr{M}$, then there exists a bijection $\varphi$ between $\left\{[\mathscr{J}]_{G} \mid \mathscr{J}\right.$ is an $\mathscr{N}$-injector of $L(G)\}$ and $\left\{[\mathscr{V}]_{G} \mid \mathscr{V}\right.$ is an $\mathscr{N}$-injector of $\left.G\right\}$ given by

$$
\varphi\left([\mathscr{J}]_{G}\right)=[\mathscr{V}]_{G},
$$

where $\mathscr{V}$ is an $\mathcal{N}$-injector of $G$ that contains $\mathscr{J}$.

Proof. Let $I_{1}, I_{2}$ be $\mathcal{N}$-injectors of $L(G)$ which are conjugate in $G$, i.e. $I_{2}=I_{1}^{g}$ for some $g \in G$. Let $V_{1}, V_{2}$ be $\mathscr{N}$-injectors of $G$ that contain $I_{1}, I_{2}$, respectively. Then $I_{2}=I_{1}^{g} \leqslant V_{1}^{g}$. Therefore $I_{2} F(G) \leqslant V_{1}^{g} \cap V_{2}$, and by Lausch's Theorem, $V_{1}^{g}$ and $V_{2}$ are conjugate in $G$.

Obviously $\varphi$ is surjective. Moreover, if $I_{1}, I_{2}$ are $\mathcal{N}$-injectors of $L(G)$ such that

$$
\varphi\left(\left[I_{1}\right]_{G}\right)=\left[V_{1}\right]_{G}=\left[V_{2}\right]_{G}=\varphi\left(\left[I_{2}\right]_{G}\right),
$$

then there exists $g \in G$ such that $V_{2}=V_{1}^{g}, I_{2}=V_{2} \cap L(G)=V_{1}^{g} \cap L(G)=$ $\left(V_{1} \cap L(G)\right)^{g}=I_{1}^{g}$.

Set $S=\left\{[V]_{G} \mid V\right.$ is an $\mathcal{N}$-injector of $\left.G\right\}$.

COROLlaRY. The following conditions are equivalent:

(i) $G$ is an $\mathscr{M}$-group, and $|\mathscr{S}|=1$;

(ii) $G$ is an $\mathscr{N}$-constrained group.

Proof. (ii) $\Rightarrow$ (i). By Proposition 1, we know that all $\mathscr{N}$-constrained groups are $\mathscr{M}$-groups. A. Mann's theorem [6] then implies that $|S|=1$.

(i) $\Rightarrow$ (ii). We must prove that $L(G)=1$ [4]. Let $P, Q$ be $p$-Sylow and $q$-Sylow subgroups of $L(G)(p \neq q)$, respectively, and let $I_{1}, I_{2}$ be $\mathscr{N}$-injectors of $L(G)$ that contain $P, Q$, respectively. Let $V_{1}, V_{2}$ be $\mathcal{N}$-injectors of $G$ such that $I_{1} \leqslant V_{1}$ and $I_{2} \leqslant V_{2}$. Then there exists $g \in G$ such that $V_{1}^{g}=V_{2}$, and so $I_{2}=I_{1}^{g}$. Therefore $P^{g} \leqslant I_{1}^{g}=I_{2}$. This argument shows that $\left|I_{2}\right|=|L(G)|$, and so $L(G)$ is nilpotent. Hence $L(G)=1$.

\section{References}

[1] B. Fischer, W. Gaschütz and B. Hartley, 'Injektoren endlicher auflösbarer Gruppen', Math. Z. 102 (1967), 337-339.

[2] D. Gorenstein and J. Walter, 'The $\pi$-layer of a finite group', Illinois J. Math. 15 (1971), $555-564$.

[3] B. Huppert and N. Blackburn, Finite Groups III (Springer-Verlag, Berlin, 1982). 
[4] M. J. Iranzo and F. Perez Monasor, 'Existence of $\mathrm{N}$-injectors in a not central normal Fitting class', Israel J. Math. 48 (1984), 123-128.

[5] H. Lausch, 'Conjugacy classes of maximal nilpotent subgroups', Israel J. Math. 47 (1984), 29-31.

[6] A. Mann, 'Injectors and normal subgroups of finite groups', Israel J. Math. 9 (1971), 554-558.

\section{Departamento de Algebra y Fundamentos}

Facultad de Ciencias Matemáticas

Universidad de Valencia

Valencia

Spain 